ÉGYPTE monde arabe

\section{Égypte/Monde arabe}

$11 \mid 1992$

À propos de la nationalité

\title{
Le monopole national d'exercice d'une profession libérale : le cas de la médecine
}

\section{Sylvia Chiffoleau}

\section{(2) OpenEdition}

\section{Journals}

Édition électronique

URL : https://journals.openedition.org/ema/311

DOI : 10.4000/ema.311

ISSN : 2090-7273

\section{Éditeur}

CEDEJ - Centre d'études et de documentation économiques juridiques et sociales

\section{Édition imprimée}

Date de publication : 30 septembre 1992

Pagination : 59-76

ISSN : 1110-5097

\section{Référence électronique}

Sylvia Chiffoleau, «Le monopole national d'exercice d'une profession libérale : le cas de la médecine », Égypte/Monde arabe [En ligne], 11 | 1992, mis en ligne le 08 juillet 2008, consulté le 07 juillet 2022. URL : http://journals.openedition.org/ema/311 ; DOI : https://doi.org/10.4000/ema.311

Ce document a été généré automatiquement le 7 juillet 2022.

Tous droits réservés 


\title{
Le monopole national d'exercice d'une profession libérale : le cas de la médecine
}

\author{
Sylvia Chiffoleau
}

1 La mise en place progressive d'une nationalité égyptienne dans les premières décennies $\mathrm{du} \mathrm{xx}^{\mathrm{e}}$ siècle s'articule au processus de fabrication d'une identité professionnelle alors en cours du corps médical égyptien. Face aux, praticiens étrangers installés dans le pays et face aux autorités coloniales, celui-ci cherche en effet à affirmer son autonomie à travers la création de nouvelles institutions nationales: Faculté de médecine, ministère de la Santé et syndicat professionnel.

Depuis un siècle déjà les médecins égyptiens sont détenteurs d'un savoir intellectuel et technique spécifique hérité de l'Occident; en acquérir le monopole de la pratique dans leur propre pays leur permettrait d'assumer cet héritage et de le transformer en filiation plutôt qu'en simple transfert. D'autre part, ils cherchent alors à s'affirmer comme catégorie socio-professionnelle compétente et apte à définir un projet social adapté au cas particulier de l'Égypte. Ainsi, ils commencent à dénier aux étrangers la capacité de comprendre la société égyptienne et ses besoins médicaux, notamment la nécessité de se consacrer au développement rural jusqu'alors largement négligé. Enfin, se constituer en groupe social représentatif, s'appuyant sur ses propres institutions, offrirait aux médecins égyptiens la possibilité d'obtenir un accès au pouvoir et d'intervenir sur les politiques publiques en matière de santé.

La question de la nationalité traverse ainsi certains problèmes spécifiques à la profession médicale que l'on cherche à résoudre durant cette même période. I! s'agit dans un premier temps de définir les critères d'une compétence professionnelle, c'està-dire de faire admettre la valeur du diplôme égyptien et celle de l'institution qui le délivre. Ensuite, il convient d'imposer le principe selon lequel à qualification égale, on peut prétendre à une fonction égale. Enfin, il reste à achever le processus d'institutionnalisation de la profession par la création d'un organe disciplinaire.

Le droit d'exercer la profession médicaleL'École de médecine du Caire 
4 La formation des médecins est assurée depuis longtemps en Égypte même, puisque l'École de médecine du Caire, première institution de ce type dans le monde arabe, est créée dès 1827. Face au dépérissement de la médecine arabe classique, Muhammad Ali fait le choix d'importer la science médicale occidentale afin de lutter contre les maladies qui déciment une armée sur laquelle il compte appuyer sa puissance.

5 Les premières générations de médecins égyptiens formés à l'École du Caire sont recrutées dans les classes les plus pauvres, souvent parmi les orphelins, dans les kuttâb (écoles coraniques) ou à l'Université d'al-Azhar. Au terme de quatre années d'études, ils sont affectés à l'armée avec un rang militaire et le titre d'officier de santé ${ }^{1}$ puis, à partir de 1842 , également dans un service civil encore très restreint, destiné à répandre la vaccination antivariolique dans l'ensemble du pays ainsi que quelques règles élémentaires d'hygiène.

6 Dans les années 1860, la fonction publique - qui accueille la majorité des praticiens formés au Caire- commence à acquérir un certain prestige ${ }^{2}$. Désormais, l'École de médecine recrute dans toutes les classes de la société; les notables locaux et les fonctionnaires ne répugnent plus à y envoyer leurs fils. Certains médecins issus de la première période assurent l'enseignement à l'École, celle-ci fonctionnant de façon autonome et sans apport étranger de 1863 à 1876, sous la direction de Muhammad alBaqIi, le plus jeune et le plus brillant des étudiants envoyés en France en 1832 pour y compléter leur formation.

7 L'occupation anglaise vient mettre un terme à cette autonomie. Un directeur britannique est nommé à la tête de l'École en 1886 et, en 1893, l'ensemble des chaires d'enseignement sont de nouveau détenues par des étrangers. En 1887, les autorités anglaises réforment le système d'enseignement de l'École de médecine. Ne sont plus admis que ceux qui possèdent !e diplôme d'études secondaires récemment institué, ce qui l'imite aux Égyptiens et aux étrangers installés en Égypte ${ }^{3}$ l'accès à l'École. La scolarité .devient payante et le recrutement se resserre autour des membres des classes supérieures. Le diplôme de docteur en médecine est enfin délivré moyennant le soutien d'une thèse après cinq années d'études.

8 Cependant, si ces réformes témoignent d'une volonté d'aligner la formation dispensée au Caire sur les critères internationaux de qualification, les Anglais n'en limitent pas moins sévèrement le rôle dévolu aux médecins. Ainsi, pendant toute la période du Protectorat, un numerus clausus ferme est imposé à l'École. Malgré la délivrance d'un diplôme de doctorat de médecine, les Anglais maintiennent de fait un statut d'officier de santé pour les praticiens issus de l'École du Caire. En l'absence de toute politique globale de santé, les besoins en personnel médical sont en effet limités et la vocation des médecins circonscrite à la surveillance de l'état de santé des populations. Tous les médecins exerçant en Égypte sont ainsi «tenus de dénoncer à l'autorité sanitaire, sur la formule imprimée que la poste délivre gratis, les cas de maladies contagieuses qu'ils auront constatées et qui seraient de nature à engendrer une épidémie »" Aux yeux des Anglais, la réalisation d'une telle tâche, qui laisse peu de place à la pratique de la médecine et au soulagement de la souffrance, ne nécessite pas plus de dix ou vingt diplômés chaque année. Il faudra attendre 1923 et le retour à une certaine autonomie de l'Égypte pour que le nombre des diplômés atteigne une quarantaine par an.

9 Cette volonté de limiter le développement de la profession médicale égyptienne incite également les Anglais à s'opposer à la création de cycles supérieurs d'études de spécialités à l'École de médecine du Caire. Cette carence encourage les familles 
égyptiennes aisées à financer pour leurs enfants des études post-doctorales dans les Facultés européennes; ceux qui en reviennent munis de diplômes de spécialisation peuvent obtenir des postes de professeurs-assistants à l'École de médecine.

La politique menée par les autorités britanniques, qui vise à maintenir les praticiens égyptiens dans une position subalterne, conduit finalement à l'émergence d'une élite médicale locale qui investit peu à peu certains postes d'enseignement, ainsi que la pratique hospitalière et privée. Cette fraction de la profession médicale égyptienne conquiert rapidement un certain prestige social puisqu'elle compte dans ses rangs de nombreux Beys et quelques Pachas, parmi lesquels le Docteur Ali Ibrahim dont l'action en faveur de l'égyptianisation du corps médical va dominer la première moitié du XXe siècle.

Les médecins étrangers

11 Cette nouvelle élite qui cherche à asseoir définitivement sa position sociale et scientifique subit la concurrence des médecins étrangers, fort nombreux en Égypte. La présence de ceux-ci est anciennement attestée puisque dès la fin du XVI ${ }^{\mathrm{e}}$ siècle, Prosper Alpin $^{5}$ signale des médecins de consulat, surtout vénitiens, qui se consacraient à la pratique de clientèle auprès de leurs concitoyens plus confiants en une médecine qu'ils connaissaient, même si celle-ci n'était guère plus performante, à cette époque, que la médecine autochtone.

Le XIXe siècle attire dans le pays de nombreux médecins étrangers qui s'installent surtout en cabinet privé alors que les praticiens formés au Caire sont en majorité employés dans le service public. Leur nombre augmente de façon très importante après le boom cotonnier de 1860 puis avec l'occupation anglaise ${ }^{6}$. Si la clientèle solvable est restreinte, les occasions de faire fortune dans cette profession ne sont pas négligeables, au point qu'aventuriers, escrocs et autres médicastres sans diplôme tentent également leur chance.

13 Cette situation est favorisée par l'absence de contrôle sur l'exercice de la profession Jusqu'au décret du 13 Juin 1891. Celui-ci constitue une première étape dans ce sens, mais il ne prévoit aucune limitation pour les étrangers. La nouveauté tient au fait qu'il faut désormais produire un authentique diplôme de médecine délivré par une faculté reconnue, de quelque pays qu'elle soit, ceci pour supprimer les fraudes et la pratique illégale de la profession. L'autorisation d'exercer la médecine, délivrée par l'Administration de l'Hygiène publique et confirmée par une publication au Journal Officie!, ne peut être obtenue que sur présentation des titres. Cette exigence nouvelle correspond également à une évolution générale du corps médical puisqu'au cours de la même période en Occident, les médecins se voient reconnaître définitivement le monopole de leur savoir?.

La primauté du diplôme de la Faculté de médecine du Caire

14 La première Guerre Mondiale offre à la nouvelle élite médicale égyptienne l'opportunité de s'affirmer professionnellement et de commencer à s'émanciper de la tutelle des Britanniques. Les professeurs-assistants de l'École occupent pour quelques années les chaires abandonnées par les Anglais mobilisés sur le front et les médecins employés aux postes subalternes de la fonction publique prennent à leur charge les responsabilités des inspecteurs divisionnaires. Profitant de ce contexte favorable, Ali Ibrahim crée en 1917 le Journal de l'Association médicale égyptienne ${ }^{8}$, qui se fera le porte-parole des tentatives d'autonomisation du corps médical égyptien au cours des années suivantes. L'offensive est menée prioritairement en direction de l'École de 
médecine où Ali Ibrahim obtient la création des deux premières chaires égyptiennes au début des années vingt. Lui-même est élu vice-doyen de la Faculté de médecine en 1926 puis doyen - il est le premier Égyptien à occuper ce poste - en 1929. Faisant suite à la loi de 1923 sur l'égyptianisation des offices publics et à celle de 1926 sur la nationalité égyptienne ${ }^{9}$, un nouveau règlement pour l'exercice de la profession médicale, remplaçant celui de 1891, est adopté en 1928. Cette seconde législation favorise « l'égyptianité » naissante puisqu'elle stipule que, pour exercer la profession médicale en Égypte, il faut être muni du diplôme délivré par la Faculté de médecine de l'Université du Caire, qui accueille quasi-exclusivement des étudiants égyptiens, et être inscrit auprès de l'Administration de l'Hygiène publique. Les médecins diplômés à l'étranger doivent passer une épreuve basée sur le programme de l'examen final de la Faculté de médecine du Caire où ils peuvent utiliser l'une ou l'autre des langues juridiques employées devant les Tribunaux mixtes d'Égypte. En cas de réussite, ils sont ensuite invités à s'inscrire auprès de l'Administration de l'Hygiène publique. Seuls les professeurs des Facultés étrangères et " les médecins ayant exercé pendant une période de cinq ans au moins dans un hôpital reconnu par le gouvernement égyptien parmi les grands hôpitaux étrangers $»^{10}$, personnes dont on peut difficilement contester la compétence, sont dispensés de cet examen. En 1932, une nouvelle loi étend largement aux diplômés égyptiens des Facultés étrangères la dispense des examens, de façon à ne pas pénaliser les Égyptiens formés à l'étranger.

Cette nouvelle législation témoigne d'une volonté de la Faculté du Caire d'affirmer sa capacité à former des médecins pour l'Égypte, et de se voir reconnue comme seule compétente pour fixer les critères de qualification. Depuis 1925 en effet, la vieille École de médecine a été intégrée à l'Université égyptienne qui vient de naître; son diplôme est officiellement reconnu par l'État, qui en assure désormais le contrôle. Elle a changé de statut pour devenir une faculté, ce qui tend à lui conférer une sorte de compétence " universelle ", égale à celle des facultés de médecine occidentales. Les nouveaux 'mandarins' égyptiens, avec à leur tête Ali Ibrahim, cherchent de cette façon à assurer leur contrôle sur la reproduction du corps médical et perpétuer ainsi leur domination.

Le partage des modes de pratique

16 Une fois reconnue la valeur du diplôme égyptien et de l'institution qui le délivre, la législation va s'attacher, dans une seconde période, à lutter de façon plus explicite contre la concurrence exercée par les médecins étrangers qui, d'une part, monopolisent toujours la pratique privée à laquelle aspirent les médecins égyptiens et, d'autre part, occupent la majorité des postes de la haute fonction publique.

Les médecins de santé publique : un groupe dominé

17 La question d'une nationalité naissante soulevée par cette législation renvoie en effet à celle des fonctions offertes par l'État pour son propre service. Les médecins formés au Caire continuent d'être prioritairement employés dans le service public, ce qui bénéficie aux Égyptiens de souche, largement majoritaires à la Faculté du Caire, comme aux « sujets locaux » originaires des provinces de l'Empire ottoman. Dans un décompte de l'ensemble des praticiens exerçant en Égypte, effectué par les autorités britanniques en 1918, on retrouve ainsi sous le titre "d'Égyptiens » les sous-catégories utilisées également dans le recensement général de la population: musulmans, coptes, Syriens et Arméniens. Cette classification témoigne du maintien de l'ordre ancien qui consent certains avantages aux étrangers, en particulier un accès à la fonction publique ${ }^{11}$. 
18 Les autorités britanniques trouvent à leur arrivée en Égypte un système de santé publique déjà en fonctionnement depuis plusieurs décennies et dont ils accentuent le caractère pyramidal. A la tête du dispositif, des administrateurs anglais occupent les postes de direction dans la capitale égyptienne. Un inspecteur divisionnaire britannique assure ensuite la supervision de deux ou trois mudiriyya (gouvernorats). Sous ses ordres est placé un inspecteur égyptien pour chaque province, ce dernier contrôlant lui-même les médecins de markaz, seuls praticiens en contact réel avec la population des provinces et responsables des bureaux sanitaires installés en principe dans chaque ville principale de markaz. Les salaires des officiers de santé et médecins de quatrième et troisième classe affectés à ces postes sont très modestes, insuffisants pour leur assurer un niveau de vie conforme à leurs aspirations.

19 Le droit de cumuler leur emploi public à une pratique de clientèle, en dehors des heures d'ouverture des bureaux de santé, leur est cependant consenti, ce qui leur procure des revenus substantiels, largement supérieurs à leur salaire de fonctionnaire. Ceci explique sans doute aussi pourquoi on ne trouve pas ou peu de médecins dans des localités de taille inférieure aux villes de markaz, où la population est suffisamment nombreuse et diversifiée pour fournir cette clientèle. Grâce au prestige lié à la maitrise d'un savoir moderne d'une part, et aux revenus qu'ils peuvent acquérir par la pratique en cabinet privé d'autre part, les médecins de markaz parviennent ainsi à occuper une position de notabilité locale qu'ils réinvestissent souvent dans des mandats représentatifs.

20 La liste officielle des médecins autorisés à exercer leur profession en Égypte en $1922^{12}$ permet de dégager quelques tendances de la répartition géographique des différents modes d'exercice, en fonction des pays d'origine du diplôme. Sur les 1533 médecins enregistrés à cette date, 550 seulement ont obtenu celui-ci au Caire, auxquels on peut ajouter 89 médecins sans doute Égyptiens, en possession d'un diplôme de spécialité d'une Faculté étrangère et dont la plupart ont eu le doctorat de médecine au Caire, ce qui porte la proportion des médecins diplômés en Égypte à environ 40 \%.

21 Dans leur quasi-totalité, les étrangers d'Europe ou des États-Unis pratiquent en cabinet de clientèle au Caire, à Alexandrie ou dans la zone du Canal de Suez. Certains Britanniques sont par ailleurs employés dans les postes de la haute fonction publique ou à la Faculté du Caire. La province n'attire guère que quelques Américains attachés à l'hôpital des missions américaine d'Assiout et de Tanta ou, par exemple, un Anglais employé à l'hôtel Winter de Louxor.

22 Les « sujets locaux », diplômés de Constantinople ou de Beyrouth, représentent $19 \%$ de l'ensemble des médecins en exercice. Ils sont en majorité installés en pratique privée (197 sur 289), surtout au Caire et à Alexandrie. Contrairement aux étrangers occidentaux, quelques-uns d'entre eux se sont fixés en province, y compris dans le $\operatorname{sud}^{13}$. En vertu des avantages qui leur sont accordés, certains de ces médecins originaires des provinces de l'ex-Empire ottoman occupent des postes de la fonction publique dans les bureaux de santé des villes de markaz et la plupart des médecins de l'armée affectés au Soudan sont des diplômés de la Faculté de médecine de Beyrouth.

23 La majorité des bureaux de santé des villes de markaz reste cependant occupée par des Égyptiens diplômés au Caire. Ceux-ci se voient fortement concurrencés dans leur second emploi consacré à la pratique privée par un nombre important de praticiens grecs. Ces derniers, contrairement aux ottomans, ne sont jamais employés dans la fonction publique mais sont installés en pratique de clientèle sur l'ensemble du 
territoire, jusque dans les localités les plus éloignées. Dès qu'un bourg ou une petite ville possède une population suffisante pour produire une clientèle privée, on peut trouver un ou deux, voire trois médecins grecs.

Leur présence est d'autant plus difficilement ressentie par les médecins fonctionnaires égyptiens que ceux-ci cherchent alors à revaloriser leur statut. Ils revendiquent en effet une reconnaissance sociale égale à celle accordée aux juges et aux ingénieurs dont le prestige semble plus élevé à cette époque. Dans cette quête d'une identité conciliant statut social valorisé et nécessité de pratiquer en milieu semi-rural, l'exercice privé de la profession médicale devient un atout majeur. Les nombreux refus de promotion à des postes d'inspecteurs, qui réclament des déplacements continuels privant de la possibilité d'une pratique de clientèle, témoignent de l'importance prise par cette dernière dans la vie professionnelle et sociale d'un médecin de markaz.

La haute fonction publique

La présence des médecins étrangers est particulièrement massive dans tes postes de responsabilité et au niveau supérieur de l'Administration de l'Hygiène publique. Le régime colonial favorise bien sûr cette situation, les Britanniques détenant les postes clés, mais elle bénéficie également à d'autres étrangers occidentaux, ressortissants des puissances capitulaires, en particulier les Italiens. Dès 1915 pourtant, une circulaire consacre le principe fondamental d'après lequel les emplois de l'État ne doivent être attribués qu'à des candidats «de nationalité égyptienne». L'indépendance formelle accordée à l'Égypte en 1922 donne lieu l'année suivante à une loi précisant les conditions très avantageuses de mise à la retraite ou de licenciement des agents de nationalité étrangère employés par l'État égyptien. Le service maritime et quarantenaire est explicitement exclu de cette mesure et garde ainsi son caractère d'enclave étrangère dans le système sanitaire égyptien. Appliquée à ce dernier, la loi souffre également de nombreuses exceptions. Les dérogations se multiplient, excluant les Égyptiens des charges de la haute fonction publique et témoignant du fait que les autorités d'occupation ne sont guère convaincues de la valeur et de la compétence des Égyptiens formés au Caire, malgré tous les efforts déployés dans ce sens par Ali Ibrahim.

Les postes de décision à la tête de l'Administration de l'Hygiène publique constituent l'enjeu le plus important de la lutte que se livrent Égyptiens et Britanniques. Le corps médical égyptien réclame en effet la création d'une administration compétente pour le seul domaine de la santé, dirigée par des Égyptiens mieux à même de s'imposer comme experts de la situation sanitaire du pays. La santé ne relève depuis 1886 que d'un simple département attaché au ministère de l'Intérieur. Devant l'importance croissante prise par ses services, ce département est transformé en 1920 en sous-secrétariat d'État dépendant toujours du ministère de l'Intérieur. Après la loi de 1923, le sous-secrétaire britannique cède sa place à un Égyptien, Cette concession ne vise finalement qu'à donner l'illusion d'un État indépendant puisque le cadre supérieur de l'Administration de l'Hygiène publique, qui compte douze postes, reste essentiellement aux mains des Anglais. Cette situation se maintient jusqu'en 1936, date de la création du ministère de la Santé qui marque la reconnaissance définitive de la spécificité du rôle social accordé à la médecine.

27 Par ailleurs, une double série d'arguments sert à justifier le recrutement d'étrangers aux postes supérieurs de la fonction publique. La première réclame la maîtrise des langues européennes et la familiarité avec les cultures étrangères. Les Égyptiens formés 
au Caire à la médecine moderne depuis plus de cent ans et qui comptent parmi les catégories les plus "occidentalisées » sont pourtant soupçonnés de ne pas répondre à ces critères. Ainsi, en septembre 1915, un emploi d'inspecteur sanitaire est attribué à un candidat de nationalité italienne. Sa fonction consiste à aider l'inspecteur sanitaire de la ville du Caire dans les lieux habités par les Italiens, ce qui permet d'affirmer « qu'il est indispensable de nommer un agent de la même nationalité afin qu'il puisse exécuter facilement les ordres de l'Administration ${ }^{14}$.

De même pour la nomination, en 1920, d'un inspecteur à la section des épidémies de l'Administration de l'Hygiène publique: le titulaire étant destiné à remplir les fonctions de médecin du port d'Alexandrie, "l'Administration considère qu'il est nécessaire que le candidat à choisir à cet effet soit un non-Égyptien, en raison des rapports continuels qu'il aura avec les voyageurs, pour la plupart étrangers, rapports qui exigent, en même temps qu'une certaine expérience, une connaissance suffisante de plusieurs langues étrangères $»^{15}$.

Un autre type d'argument utilisé pour appuyer la nomination d'étrangers à des postes supérieurs consiste à rappeler l'absence de formation aux spécialités médicales à la Faculté du Caire. Le problème surgit en 1919 au moment de la nomination d'un inspecteur pour les maladies vénériennes. En effet, selon l'Administration de l'Hygiène publique, «cette branche de la médecine exige des connaissances et une pratique technique spéciale et comporte à ce titre le recrutement d'un fonctionnaire ayant acquis - soit en Europe soit dans l'armée - une expérience particulière dans cette branche et qui soit capable de donner une nouvelle impulsion à cette section des services sanitaires $\aleph^{16}$. La même année, la nomination de quatre inspecteurs d'hygiène donne l'occasion de souligner que les Égyptiens ne possèdent pas ce type de spécialisation, ces titres n'étant délivrés qu'en Europe. La création de cycles d'études post-doctorales est pourtant une des revendications les plus précises du corps médical égyptien. Elle n'aboutira qu'en 1930, avec l'ouverture d'un département de médecine tropicale et d'hygiène, première spécialité officiellement reconnue en Égypte.

Ce type de difficultés et les dérogations auxquelles elles donnent lieu ne disparaîtront que dans les années quarante, au moment où les premiers diplômés sortiront des sections de spécialités créées dans les années 1930 à la Faculté du Caire. Jusqu'alors, les quelques étudiants égyptiens qui vont compléter leurs études à l'étranger et y obtiennent un diplôme post-doctoral de spécialisation sont pour la plupart nommés à des postes d'assistants à la Faculté de médecine, position qu'ils cumulent généralement avec une pratique privée de cabinet.

La revendication d'un espace de pratique privée

31 Les conditions d'exercice offertes par la fonction publique dans les postes subalternes étant très médiocres, et l'accès aux postes supérieurs très limité pour les médecins égyptiens, ceux-ci cherchent de plus en plus à investir la pratique privée, ou du moins à cumuler les deux modes de pratique. Il est vrai que la période privilégie les valeurs libérales et qu'on assiste alors, en Europe comme en Égypte, à la codification des principes de la médecine dite libérale. Cependant la pratique de clientèle est largement dominée, et depuis fort longtemps, par les médecins étrangers, ressortissants des puissances capitulaires ou de l'ex-Empire ottoman. On a vu que les Grecs étaient bien implantés en province ; le phénomène est plus massif encore dans les grandes villes du pays. 

l'année 1913, à une réunion des médecins libéraux du Caire destinée à débattre des problèmes rencontrés alors par cette fraction du corps médical. Cette réunion rassemble 170 médecins qui se désignent comme «Égyptiens ", mais on compte parmi eux la présence du docteur Comanos Pacha, médecin grec formé en Allemagne et établi en Égypte depuis 1877, où il occupe la fonction de médecin personnel du Khédive. Pour certains, la durée du séjour en Égypte semble donc octroyer des privilèges de «nationalité ». affirment qu'avec la crise économique que le pays traverse alors, les médecins éprouvent de plus en plus de difficultés à vivre de leur exercice. Si les praticiens installés au Caire depuis 20 ans ou plus, qui ne sont que $10 \%$ de l'ensemble, gardent une position excellente et parfois ont fait fortune, ceux qui pratiquent depuis 10-15 ans, soit les plus nombreux, ont assisté à une baisse sensible de leurs ressources. Quant à ceux qui exercent depuis moins de 5 ans, leur situation est considérée comme catastrophique : ils gagnent à peine le salaire en vigueur dans !a fonction publique, ce qui paraît bien dégradant aux yeux de ces médecins libéraux. La principale cause avancée pour expliquer cette dégradation de leur condition est la pléthore de médecins que compte le Caire et l'Égypte en général.

La référence à cette pléthore est une constante du discours professionnel des médecins. Bien que les effectifs soient très faibles à cette époque, les praticiens perçoivent pourtant une très forte concurrence qui menace la rente médicale, car la demande solvable est très réduite. De 1902 à 1912, rappellent les participants, le nombre total de médecins privés en Égypte a augmenté de $30 \%$ et de $46 \%$ dans la seule capitale. L'entente ne semble pas régner parmi eux, estiment-ils, et ils regrettent l'absence de confraternité au sein du corps médical. Ils mettent également en cause, sans aucunement prendre en compte la structure sociale du pays, la concurrence des œuvres gratuites et des dispensaires ouverts aux indigents. Mais le problème, selon eux, vient surtout de l'affluence des médecins étrangers et des "médecins-touristes ", célèbres praticiens occidentaux qui viennent effectuer une série d'actes médicaux grassement payés et agrémentés d'un séjour touristique au pays des pharaons. Pour lutter contre cet envahissement, les porte-parole de cette réunion proposent de «faire connaître au corps médical des pays étrangers, par des circulaires émanant du corps médical égyptien, la vraie situation des médecins débutants en Égypte et montrer les difficultés matérielles et morales que rencontrent les médecins étrangers qui voudraient s'établir -dans ce pays. Eclairés par ces circulaires, les médecins étrangers hésiteraient à venir s'y installer et l'encombrement serait moindre $»^{17}$.

Cependant, malgré ces avertissements, les médecins étrangers continuent d'affluer. La tolérance de la réglementation de 1928 sur l'exercice de la profession et le maintien du régime capitulaire encouragent leur installation en Égypte. Ainsi, à la fin des années 20 et au début des années 30, si la Faculté de médecine du Caire produit chaque année environ 90 à 100 nouveaux diplômés, l'Administration de l'Hygiène publique autorise quant à elle 150 à 200 nouveaux praticiens, la différence représentant l'apport annuel de médecins étrangers.

Le poids de ceux-ci est tel en Égypte qu'il faut attendre dix ans après la conférence de Montreux et la suppression des capitulations pour voir apparaître une nouvelle législation réglementant la pratique médicale. La loi de 1948, adoptée alors que la 
nationalité égyptienne a définitivement pris forme, limite aux seuls Égyptiens - ainsi, qu'aux ressortissants des pays dont les lois permettent à ces derniers d'y exercer la médecine - le droit de pratiquer leur profession en Égypte. Elle maintient d'autre part le principe de l'examen de tous les porteurs de diplôme étranger ${ }^{18}$.

Une nouvelle loi votée après la Révolution, en 1954, réaffirme le monopole désormais détenu par les Égyptiens sur l'exercice de la profession médicale, autant dans sa formule libérale que dans celle du service public. Cette loi précise que "nul ne peut, à titre quelconque, exercer la médecine en Égypte s'il ne remplit les trois conditions suivantes

- être de nationalité égyptienne ou être d'un pays dont les lois permettent aux Égyptiens d'y exercer la médecine ${ }^{19}$;

- être immatriculé au Registre des médecins tenu au ministère de l'Hygiène publique ;

- être immatriculé au tableau de l'Ordre des médecins $»^{20}$.

Les Égyptiens apportent la preuve de leur nationalité en produisant le certificat délivré par le ministère de l'Intérieur. Cette clause implique que les ressortissants de l'exEmpire ottoman qui désirent .exercer la médecine en Égypte, sont tenus de faire le choix de la nationalité égyptienne.

La double pression exercée par la législation sur l'autorisation d'exercer la médecine et par celle concernant plus proprement la mise en place d'une nationalité égyptienne conduit finalement à l'éviction quasi-totale des médecins étrangers. Ainsi, en 1955-5621, il ne subsiste que quelques étrangers confinés dans les hôpitaux communautaires (français, italien...) ou, pour les plus âgés, en pratique privée au Caire et à Alexandrie. Les emplois en province, autant publics que privés, sont exclusivement occupés par des Égyptiens, diplômés en Égypte ou à l'étranger, à l'exception de quelques très rares médecins âgés, diplômés de Beyrouth, qui se sont maintenus à leur poste. Les Grecs ont disparu du paysage provincial, remplacés également par des praticiens égyptiens.

Mais cette absence des étrangers signifie aussi que l'Égypte n'est plus aussi attractive et que le temps est révolu où l'on pouvait faire fortune en y pratiquant la médecine. Désormais, l'élite de la profession égyptienne détient la pratique de clientèle, nécessairement réduite au sein d'une population peu solvable, ainsi que les postes académiques. La Faculté du Caire n'est plus seule à former des médecins, deux autres Facultés ont vu le jour à 'Abbasseya et Alexandrie, produisant ensemble 3 à 400 médecins par an. Dans une société qui offre peu d'opportunités pour la pratique privée, se pose alors de nouveau le problème de la vocation sociale et professionnelle de ces médecins. La Révolution tentera de le résoudre en mettant en place le premier projet de médicalisation globale du pays. Il prendra la forme, à partir de 1962, d'un réseau rural de santé publique de première ligne qui s'appuiera sur les médecins égyptiens formés dans ces Facultés nationales.

Le contrôle de la professionLa médecine : une profession libérale

On distingue une profession d'un simple métier en ce qu'elle a acquis le monopole de $l^{\prime}$ exercice de sa fonction et le droit d'exercer son contrôle sur son propre travail ${ }^{22}$. Pour y parvenir, le groupe professionnel doit, d'une part. mettre en place des critères de qualification sanctionnés par un diplôme qui garantit une capacité technique. D'autre part, il lui revient de créer, en accord avec l'État, une institution gardienne d'un tableau professionnel sur lequel ne peuvent s'inscrire que les détenteurs du diplôme remplissant les conditions légales et morales requises. Cette institution définit la déontologie du groupe et en surveille l'application, en ce sens elle est garante de la moralité de la profession. Selon les pays, ce rôle est dévolu aux ordres professionnels 
ou à des associations médicales, alors que la fonction des syndicats est limitée à la défense des intérêts de ses membres. Cet organe de contrôle reçoit le pouvoir de sanctionner les infractions de ses membres aux règles de la déontologie et de l'exercice de la profession et constitue donc une véritable juridiction. Par ailleurs, en raison du monopole que les médecins entendent avoir sur leur savoir, ils se considèrent euxmêmes comme mieux préparés que des magistrats et seuls aptes finalement à apprécier et sanctionner certains errements professionnels. La sauvegarde de l'indépendance, de l'honneur et de la dignité de la profession se joue au sein même du groupe et ne doit pas être exposée au grand jour. Dans ce type de juridiction, les médecins sont donc jugés par leurs pairs.

Dans le contexte égyptien qui connaît longtemps la pluralité juridique, la mise en place d'une telle institution pose bien sûr problème. Même si la dimension disciplinaire ne concerne que les actes qui ne relèvent pas du droit pénal, elle se heurte à la multiplicité des nations représentées dans le corps médical égyptien, qui chacune possède ses propres modalités de contrôle. D'autre part, en Égypte, les ressortissants étrangers, quelle que soit leur profession, doivent rendre compte de leurs actes devant les juridictions consulaires ou les Tribunaux mixtes, La création d'un organe de contrôle sous la forme d'un ordre ou d'une association viendrait se superposer au système des capitulations et le perturber. L'instauration d'une juridiction professionnelle pour les médecins a donc souvent été renvoyée alors que les avocats, par exemple, en majorité égyptiens et peu concurrencés professionnellement par des étrangers, possèdent un Barreau dès 1912.

Les premières tentatives de création d'une organisation professionnelle

La profession médicale, toujours prompte à militer pour la défense de ses propres intérêts, s'est très tôt mobilisée dans le but de créer une 'organisation professionnelle. Les premières tentatives témoignent de la volonté d'opérer une synthèse des différentes nationalités au sein d'une institution unique. Ainsi, en novembre 1910, une expérience visant à former un organe représentatif mixte avorte en raison de la diversité des nationalités et des religions en présence. Elle aboutit finalement à un partage au sein de deux institutions: l'Association médicale internationale, qui regroupe les médecins étrangers, et un Syndicat médical d'Égypte réservé aux praticiens égyptiens. Quoiqu'il en soit, ces deux organisations n'ont d'existence que nominale et ne produisent aucune œuvre d'utilité professionnelle. Ali Ibrahim tente de nouveau l'unification de ces deux syndicats pendant la guerre mais il se heurte aux mêmes blocages et doit y renoncer. Ce nouvel échec rend compte de la diversité des origines, des formations, des modes de pratique mais surtout de l'impossibilité de s'entendre sur les modalités du contrôle de la profession.

Les difficultés rencontrées dans l'application du règlement sur l'exercice de la pharmacie, édicté en 1891, illustrent bien les contradictions résultant du régime des capitulations, qui empêche le gouvernement égyptien d'effectuer les actes juridiques pourtant nécessaires à un contrôle efficace des professions libérales. L'article 7 de cette loi prévoyait en effet l'inspection périodique, par l'Administration de l'Hygiène publique, « des pharmacies civiles établies en Égypte, afin de s'assurer de la bonne qualité des médicaments qui y sont débités au publie, ainsi que de ceux qui sont conservés en magasin "23. Dans le cas où l'inspection s'appliquait à une pharmacie européenne, l'Administration devait aviser le consulat concerné du jour et de l'heure fixés pour la visite. En cas d'absence du délégué consulaire, l'Administration se réservait le droit de passer outre 
et d'effectuer malgré tout l'inspection. Pour une profession composée en majorité d'étrangers, c'était accorder trop de prérogatives au gouvernement local. La pression diplomatique exercée par les puissances capitulaires aboutit finalement à la suppression des clauses relatives à l'inspection. Ainsi, à cette époque, quelle que soit l'impéritie d'un médecin, d'un dentiste ou d'un pharmacien étranger pratiquant en Égypte, elle ne peut entraîner sa disqualification, à moins que l'autorité qui l'a muni de son diplôme ne soit amenée à le lui retirer.

Une issue à cette impasse semble se dessiner lorsque l'on envisage la suppression des capitulations en 1918. A cette occasion, l'Administration de l'Hygiène publique soumet au président de la "Commission d'étude des réformes que comporte la disparition éventuelle des capitulations" une proposition pour la création d'un Ordre des médecins. Tel qu'il est prévu dans ce projet, l'Ordre doit rassembler tous les médecins autorisés à pratiquer la médecine en Égypte, aussi longtemps que leur nom reste inscrit sur le registre, et doit être organisé par nationalité. Chaque nationalité ayant au moins quinze représentants dans le corps médical peut former une section de l'Ordre. Toute nationalité qui a moins de quinze médecins doit s'allier avec une autre afin de former une section. Le conseil de l'Ordre est ensuite constitué de deux délégués élus par et au sein de chaque section,

Celte proposition n'aura pas de suite dans l'immédiat et il faudra de toute façon attendre 1937 pour assister à la suppression effective des capitulations. Le principe retenu d'une représentation par collège de nationalité ne pouvait qu'être inacceptable pour la majorité égyptienne du corps médical. Par ailleurs, cet Ordre avait vocation d'élaborer une réglementation sur l'exercice de la profession et surtout d'abriter un conseil disciplinaire dont la juridiction s'étendrait à l'ensemble des membres du corps médical, quelles que soient tes origines nationales. Cette mesure heurte cette fois les ressortissants étrangers qui ne peuvent admettre l'existence d'une institution concurrençant leurs propres juridictions, où ils savent pouvoir trouver quelque indulgence en cas de faute.

47 A la suite de ce nouvel échec, Ali Ibrahim fonde l'Association médicale égyptienne, encore vivante aujourd'hui, dont la première réunion se tient le 16 janvier 1920 et rassemble 42 médecins égyptiens. Son statut d'association et de société savante la prive d'une dimension disciplinaire, mais parmi les trois objectifs prioritaires ${ }^{24}$ qu'elle s'assigne figure l'élaboration d'un Code de déontologie, ce qui lui permet de jouer un rôle de relais jusqu'à ce que des circonstances plus favorables autorisent la création d'un Ordre.

Le Syndicat médical égyptien

II faut attendre 1940 pour que ces circonstances soient réunies et permettent l'établissement du Syndicat des médecins qui remplit la double fonction d'organe de contrôle et de syndicat professionnel. C'est encore Ali Ibrahim qui en est l'initiateur puisqu'il profite de son bref passage à la tête du ministère de la santé à partir de juin 1940 pour faire adopter la loi instituant ce syndicat ${ }^{25}$. Celui-ci apparaît comme la conclusion d'un processus d'institutionnalisation et d'émancipation d'une profession médicale qui s'est voulue de plus en plus égyptienne. Elle possède alors une Faculté nationale ancienne et performante, dirigée par un establishment autochtone qui en contrôle le recrutement. Elle a créé de nombreux journaux professionnels et scientifiques qui véhiculent les avancées de la recherche comme les valeurs de la 
profession. Enfin, elle est forte d'effectifs en augmentation constante et où la composante égyptienne est désormais majoritaire.

La création du syndicat soumet le droit d'exercer la médecine en Égypte à une double autorisation : celle du ministère de l'Hygiène publique, qui existe depuis longtemps, et celle du Syndicat. Ce dernier, et c'est en cela que réside la nouveauté, s'il peut accorder une autorisation, est également habilité à la retirer. En effet, le syndicat abrite un conseil disciplinaire autorisé à juger les entorses de ses membres au Code de déontologie. Les peines encourues vont de l'avertissement à la radiation du registre du ministère de l'Hygiène publique et du tableau de l'Ordre. Cette juridiction consacre le principe du contrôle de la profession sur ses membres et assure par là même l'autonomie du groupe. Une telle situation n'est rendue possible que par la perte des avantages jusqu'alors accordés aux étrangers. La naissance du Syndicat a ainsi dû attendre la signature des accords de Montreux, et la rédaction du code de déontologie ne s'est achevée qu'en 1949, date de la liquidation définitive du système des capitulations. Par ailleurs, cette nouvelle institution sert également de courroie de transmission entre l'État et la profession, ce qui permet à cette dernière d'avoir désormais part aux décisions politiques en matière de santé.

\section{NOTES}

1.Malgré les efforts déployés par clôt Bey pour former des médecins égyptiens d'un niveau équivalent à leurs homologues occidentaux, il ne s'est jamais résolu à leur octroyer le litre de docteur en médecine.

2.AlleaumE Ghislaine, « Naissance du fonctionnaire », Égypte. Recompositions, Peuples méditerranéens n 41-42, octobre 1987-mars 1988, pp. 67-86.

3. Ceux-ci préfèrent généralement les Écoles confessionnelles aux écoles gouvernementales. Pour s'aligner sur ces dernières, elles proposent également un diplôme d'études secondaires à partir de 1911.

4.Article 6 de l'arrêté du ministère de l'Intérieur portant règlement sur l'exercice de la médecine, en date du 13 juin 1891.

5.La médecine des Égyptiens par Prosper Alpin, 1581-1584. Le Caire, IFAO, 1980.

6.Le Conseil maritime et quarantenaire, créé en 1831 el qui ne cesse de se développer tout au long de ce XIX ${ }^{e}$ siècle, constitue quant à lui un domaine quasi-exclusivement réservé aux médecins étrangers.

7.Pour la France, voir Léonard Jacques, La médecine entre les pouvoirs et les savoirs, Aubier Montaigne, Paris. 1981 ; pour l'Angleterre, Peterson Jeanne, The médical profession in Mid-Victorian London. University of California Press. 1978.

8.L'association elle-même est restée virtuelle jusqu'en 1920, date à laquelle elle débute ses activités (cf. infra\}.

9.Le Gall-Kazazian Anne et Abecassis Frédéric, «L'identité au miroir du droit : le statut des personnes en Égypte (fin XIX - milieu XXe) ", dans ce même numéro d'Égypte/Monde arabe.

10.Décret-loi $n^{\circ} 66$ de 1928 sur l'exercice de la profession de médecin.

11.Cf. Le Gall-Kazazian et Abecassis, op. cit.

12. Cette liste semble incomplète puisqu'elle ne recense que 1533 médecins alors que celle de 1918 en comptait 2.488 . 
13.Le déséquilibre régional est alors particulièrement criant : seuls $10 \%$ du corps médical exercent en Moyenne et Haute-Égypte.

14. Archives Nationales Égyptiennes (ENA), Conseil des ministres, ministère de l'Intérieur, Affaires du personnel (carton $\mathrm{z}, 6,2$ ).

15.ENA, carton $z, 6,2$.

16.ENA, carton $\mathrm{w}, 6,2$.

17. La Presse médicale d'Égypte $\mathrm{n}^{\circ}$ 3, février 1913.

18. L'année suivante, une nouvelle loi étend largement aux Égyptiens la dispense de cet examen.

19.C'est le cas de l'Allemagne, l'Arabie Saoudite, la Grèce, l'Irak, l'Iran, l'Italie, la Jordanie et le Soudan. Les Britanniques peuvent également être autorisés à condition d'être titulaires du diplôme égyptien.

20.Loi ${ }^{\circ} 415$ du 22 Juillet 1954 réglementant l'exercice de la profession médicale.

21.Cf. Annuaire médical égyptien 1955/56, Editions Fischer, Le Caire

22.Freidson Eliot, La Profession médicale, Paris, Payot, 1984.

23.Arrêté du ministre de l'Intérieur portant règlement relatif à l'exercice de la pharmacie civile en date du 13 Juin 1891.

24.Les deux autres objectifs sont le développement de la langue arabe et son adaptation à la science médicale ainsi que la recherche orientée vers les pathologies spécifiquement égyptiennes (maladies endémiques parasitaires).

25. Nous choisissons d'utiliser le terme général de syndicat pour traduire le mot arabe niqaba. Cependant, celui-ci recouvre également la notion d'« Ordre ». Alors qu'un syndicat professionnel peut être librement constitué par toute personne exerçant cette profession, un Ordre (ou tout autre institution chargée du contrôle de la profession) ne peut exister qu'en vertu d'une loi.

INDEX

Mots-clés : histoire, médecine, professions, santé

\section{AUTEUR}

\section{SYLVIA CHIFFOLEAU}

CEDEJ 